\title{
ON EQUILIBRIUM ALLOCATIONS AS DISTRIBUTIONS ON THE COMMODITY SPACE*
}

\author{
Sergiu HART \\ Department of Mathematics, Tel Aviv University, Tel Aviv, Israel \\ Werner HILDENBRAND \\ Department of Economics, University of Bonn, Bonn, Germany \\ Elon KOHLBERG \\ Department of Economics, Harvard University, Cambridge, Mass., U.S.A. \\ Received October 1973

\begin{abstract}
It is shown that the distribution of agents' characteristics is a concise and accurate description of an economy as far as Walrasian equilibrium analysis for large economies is concerned: Let $\mathscr{E}$ be an exchange economy; $W(\mathscr{E})$, the set of Walras allocations for $\mathscr{E}$; and $\mathscr{D} W(\mathscr{E})$, the set of distributions on the commodity space of the allocations in $W(\mathscr{E})$. It is shown that for two atomless economies $\mathscr{E}_{1}$ and $\mathscr{E}_{2}$ which have the same distribution of agents' characteristics, the sets $\mathscr{D} W\left(\mathscr{E}_{1}\right)$ and $\mathscr{D} W\left(\mathscr{E}_{2}\right)$ have the same closure. For every distribution $\mu$ of agents' characteristics is defined a standard representation $\mathscr{E}^{\mu}$, and it is shown that $\mathscr{D} W\left(\mathscr{E}^{\mu}\right)$ is closed. Further, the correspondence $\mu \mapsto \mathscr{D} W\left(\mathscr{E}^{\mu}\right)$ is shown to be upper hemicontinuous.
\end{abstract}

\section{Introduction}

An cconomy $\mathscr{E}$ is usually described by a finite set $A$, the set of economic agents and by specifying for every individual agent $a$ in $A$ his characteristics. For example, in a situation of pure exchange an economic agent is characterized by his preference relation $\precsim$ and his initial endowment vector $e$. Thus an exchange economy is described by a mapping

$$
\mathscr{E}: A \rightarrow \mathscr{P} \times R_{+}^{l},
$$

when $\mathscr{P}$ denotes the set of all preference relations $\precsim$ defined on the closed positive orthant $R_{+}^{l}$ of the commodity space $R^{l}$.

If the set $A$ of agents is small (e.g., $\# A=3$ ), then the description of an economy as an assignment $a \mapsto\left(\lesssim, e_{a}\right)$ of agents to characteristics seems to be the appropriate one. However, when the set $A$ is large (e.g., $\# A>1000$ ) one is tempted to give up this individualistic description and to replace it by a more

*The essential part of the research of this paper was done during the Workshop on Mathematical Economics, University of Bielefeld, Rheda, W. Germany, June-July 1973. 
collectivistic view, namely, to consider the distribution $\mu_{\mathscr{E}}$ of the mapping $\mathscr{E}$. That is to say, for every subset $B$ in $\mathscr{P} \times R^{l}$ we consider the number $\mu_{\delta}(B)$ which is the fraction of the totality of agents in $A$ having their characteristics in the set $B$ :

$$
\mu_{\mathscr{E}}(B)=\#\{a \in A \mid \mathscr{E}(a) \in B\} / \# A .
$$

When we describe an economy by its distribution $\mu$ of agents' characteristics, then we can no longer speak of some particular agent and his particular characteristics; the set of agents is no longer specified. However, we can speak meaningfully of the fraction of the totality of agents having their characteristics in a particular subset $B$ in $\mathscr{P} \times R^{l}$.

Now the question arises: Is it legitimate to describe an economy by a distribution of agents' characteristics, that is to say, does the distribution $\mu_{\varepsilon}$ of an economy $\mathscr{E}: A \rightarrow \mathscr{P} \times R_{+}^{l}$ contain the relevant information?

The answer to this question clearly depends on what type of equilibrium concepts and what kind of problems we want to investigate. For example, if an economy is described by a distribution of agents' characteristics only, then it is not clear how the core of that economy should be defined. The concepts of 'coalition' and 'to improve upon' require the individualistic description of an economy as a mapping which assigns to every individual agent his characteristics. Yet, when we consider distribution of agents' characteristics we have large economies in mind, and for large economies we have the well-known Theorem of Aumann (1964) which says that the core and the set of Walras allocations coincide. Thus, in situations where distributions of agents' characteristics seem to be the natural concept, namely, in large economies, we can concentrate on Walras equilibria.

We shall show in what follows that with respect to Walrasian Equilibrium Theory the distribution of agents' characteristics actually contains the relevant information.

\section{Large economies}

Firstly, we have to recall the precise definition of a 'large' exchange economy.

Let $\mathscr{P}$ denote the set of preference relations $\lesssim$ on $R_{+}^{l}$ (i.e., $\lesssim$ is a reflexive, transitive complete and continuous binary relation) and $\mathscr{P}_{\text {mo }}$ is the set of monotonic preference relations (i.e., $x \leqq y, x \neq y$ implies $x<y$ ). We endow the set $\mathscr{P}_{\text {mo }}$ with a metric, the metric of closed-convergence [for details, see Kannai (1970) and Hildenbrand (1970 or 1974)]. A distribution on $\mathscr{P}_{\text {mo }} \times R_{+}^{l}$ then means a probability measure on the $\sigma$-algebra $\mathscr{B}\left(\mathscr{P}_{\text {mo }} \times R_{+}^{l}\right)$ of Borel sets in $\mathscr{P}_{\text {mo }} \times R_{+}^{l}$.

An atomless economy $\mathscr{E}$ is defined as a measurable mapping $\mathscr{E}$ of an atomless ${ }^{1}$ measure space $(A, \mathscr{A}, v)$ into $\mathscr{P}_{\text {mo }} \times R_{+}^{l}$ such that the mean endowment vector

\footnotetext{
${ }^{1}$ Measure space always means $v(A)=1$ and $v(B) \geqq 0$ for even $B \in \mathscr{A}$; a measure space $(A, \mathscr{A}, v)$ is called atomless if for every $E \in \mathscr{A}$ with $v(E)>0$ there is $S \in \mathscr{A}$ with $0<v(S)<v(E)$.
} 
$\int_{A} e \circ \mathscr{E} \mathrm{d} v$ exists and is strictly positive (e denotes the projection of $\mathscr{P}_{\mathrm{mo}} \times R_{+}^{l}$ onto $R_{+}^{l}$ ).

The distribution $\mu_{\mathscr{g}}$ of an atomless economy $\mathscr{E}$ is the image measure of $v$ with respect to the mapping $\mathscr{E}$, i.e.,

$$
\mu_{\mathscr{E}}(B)=v(\{a \in A \mid \mathscr{E}(a) \in B\})
$$

for every Borel set in $\mathscr{P}_{\mathrm{mo}} \times R_{+}^{l}$.

An integrable function $f$ of $A$ into $R_{+}^{l}$ is called a Walras allocation for economy $\mathscr{E}$ (or a competitive equilibrium) if there is a price vector $p \in R^{l}, p \neq 0$, such that

(i) a.e. in $A, f(a) \in \varphi(\mathscr{E}(a), p)$, i.e., the vector $f(a)$ is a maximal element for $\precsim_{\mathscr{E}(a)}$ in the budget-set $\left\{x \in R_{+}^{l} \mid p \cdot x \leqq p \cdot e(\mathscr{E}(a))\right\}$;

(ii) $\int f \mathrm{~d} v=\int e \circ \mathscr{E} \mathrm{d} v$, i.e., mean demand equals mean supply.

The set of all Walras allocations of the economy $\mathscr{E}$ is denoted by $W(\mathscr{E})$. The corresponding set of equilibrium prices is denoted by $\Pi(\mathscr{E})$.

It has been shown by Aumann (1966) that for every atomless economy

$$
\mathscr{E}:(A, \mathscr{A}, v) \rightarrow \mathscr{P}_{\mathrm{mo}} \times R_{+}^{l}
$$

there exists a Walras allocation. Thus the sets $W(\mathscr{E})$ and $\Pi(\mathscr{E})$ are non-empty.

\section{Equilibrium prices and distributions}

If we consider only the set $\Pi(\mathscr{E})$ of equilibrium prices of the economy $\mathscr{E}$ - not the corresponding Walras allocations $W(\mathscr{E})-$ then the situation is quite easy:

Proposition. Let $\mathscr{E}_{i}:\left(A_{i}, \mathscr{A}_{i}, v_{i}\right) \rightarrow \mathscr{P}_{\mathrm{mo}} \times R_{+}^{l}(i=1,2)$ be two atomless economies with the same distribution $\mu_{\dot{B}_{1}}=\mu_{\varepsilon_{2}}$. Then

$$
\Pi\left(\mathscr{E}_{1}\right)=\Pi\left(\mathscr{E}_{2}\right) .
$$

Proof. A price vector $p$ belongs to $\Pi\left(\mathscr{E}_{i}\right)$ if and only if $\int \boldsymbol{e} \circ \mathscr{E}_{i} \mathrm{~d} v_{i} \in$ $\int \varphi\left(\mathscr{E}_{i}(\cdot), p\right) \mathrm{d} v_{i}$, i.e., the mean supply vector belongs to the mean demand set associated with the price vector $p$. By the change-of-variable formula we have

$$
\int_{A_{i}} e \circ \mathscr{E}_{i} \mathrm{~d} v_{i}=\int_{\mathscr{I}_{\mathrm{mo}} \times R_{+}^{i}} e \mathrm{~d} \mu_{\mathscr{E}_{i}} .
$$

But also the mean demand set $\int \varphi\left(\mathscr{E}_{i}(\cdot), p\right) \mathrm{d} v_{i}$ depends only on the distribution $\mu_{\mathscr{E}_{i}}$ of $\mathscr{E}_{i}$. Indeed one has

$$
\int_{A} \varphi\left(\mathscr{E}_{i}(\cdot), p\right) \mathrm{d} v_{i}=\int_{\mathscr{F}_{\mathrm{mo}} \times R_{+}^{1}} \varphi(\cdot, p) \mathrm{d} \mu_{\mathscr{E}_{i}}
$$

since the measure space $\left(A_{i}, \mathscr{A}_{i}, v_{i}\right)$ is atomless [Hildenbrand (1974, chapter 1 , proposition 4)]. Thus the condition for $p$ to be an equilibrium price depends only on the distribution of $\mathscr{E}$, and this clearly implies the proposition. Q.E.D. 


\section{Walras allocations and distributions}

We now want to go a step further and we shall consider the equilibrium allocation as well. An allocation was defined as a mapping $f$ of the set $A$ of agents into the commodity space, i.e., an assignment of commodities to individual agents. Now, if we consider the distribution $\mu_{\varepsilon}$ of an economy $\mathscr{E}$ as the basic concept, while we regard the set of agents as not being intrinsic, we have to follow the same change of view for allocations, that is to say, we consider the distribution $\mathscr{D} f$ of the allocation $f$ in the commodity space. Then we can no longer speak of a particular commodity bundle allocated to a particular individual agent. However, given a certain subset $S$ in the commodity space, we can speak of the fraction of the totality of agents having their allocation in this set.

Unlike the case of equilibrium prices, the set $\mathscr{D} W(\mathscr{E})$ of distributions of all Walras allocations $W(\mathscr{E})$ is not completely determined by the distribution $\mu_{\mathscr{E}}$ of agents' characteristics. The following example is discussed in detail in Kannai (1970).

Example. We shall define two economies $\mathscr{E}_{1}$ and $\mathscr{E}_{2}$ with the same distribution $\mu_{\mathscr{E}_{1}}=\mu_{\mathscr{E}_{2}}$. The space of agents for both economies is the unit interval $[0,1]$ with Lebesgue measure $\lambda$. All agents, in both economies, have the same preference relation whose indifference curves are pictured in the following figure:

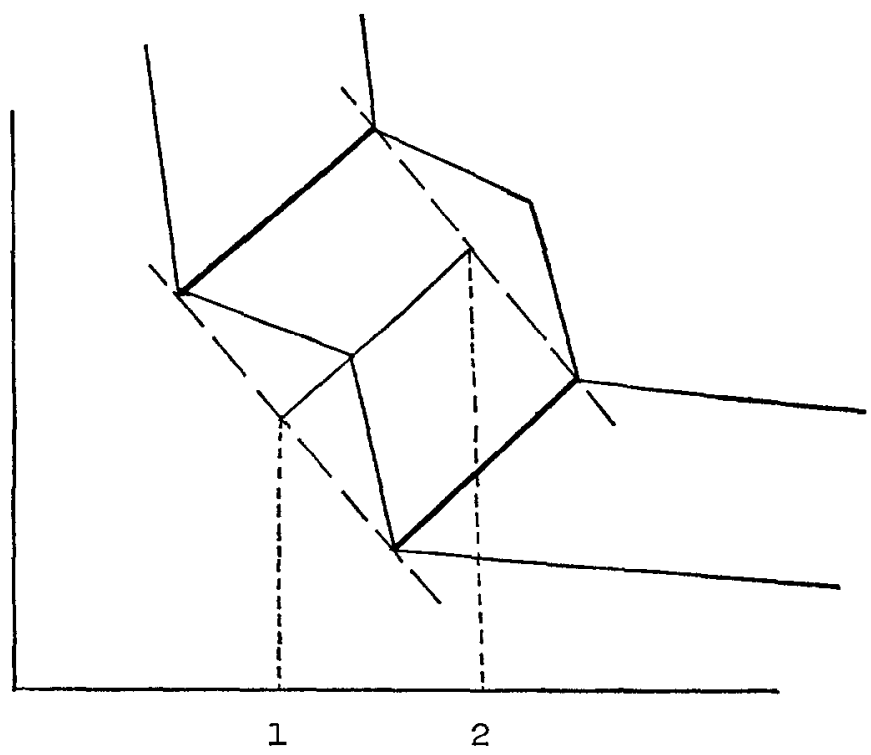

Fig. 1 
The endowments are given by

and

$$
\boldsymbol{e}\left(\mathscr{E}_{1}(t)\right)=(1+t, 1+t), \quad t \in[0,1],
$$

$$
\boldsymbol{e}\left(\mathscr{E}_{2}(t)\right)= \begin{cases}(1+2 t, 1+2 t) & \text { if } t \in\left[0, \frac{1}{2}\right), \\ (2 t, 2 t) & \text { if } t \in\left[\frac{1}{2}, 1\right] .\end{cases}
$$

One easily verifies that for the second economy $\mathscr{E}_{2}$ there is a Walras allocation $f$ such that its distribution $\mathscr{D} f$ is the uniform distribution on the darkened two segments in the above figure. However, this distribution cannot be obtained as a distribution of a Walras allocation in the first economy $\mathscr{E}_{1}$, hence

$$
\mathscr{D} W\left(\mathscr{E}_{1}\right) \neq \mathscr{D} W\left(\mathscr{E}_{2}\right) \text {. }
$$

Theorem 1. Let $\mathscr{E}_{1}$ and $\mathscr{E}_{2}$ be two atomless economies with the same distribution $\mu_{\mathscr{E}_{1}}=\mu_{\mathscr{E}_{2}}$ on $\mathscr{P}_{\mathrm{mo}} \times R_{+}^{l}$. Then the sets $\mathscr{D} W\left(\mathscr{E}_{1}\right)$ and $\mathscr{D} W\left(\mathscr{E}_{2}\right)$ of distributions of Walras allocations of $\mathscr{E}_{1}$ and $\mathscr{E}_{2}$, respectively, have the same closure with respect to the weak convergence;

$$
\overline{\mathscr{D} W\left(\mathscr{E}_{1}\right)}=\overline{\mathscr{D} W\left(\mathscr{E}_{2}\right)} \text {. }
$$

Proof. By the above Proposition we have $\Pi\left(\mathscr{E}_{1}\right)=\Pi\left(\mathscr{E}_{2}\right)$. Let $p \in \Pi\left(\mathscr{E}_{i}\right)$, and denote by $W\left(\mathscr{E}_{i}, p\right)$ the set of Walras allocations for the economy $\mathscr{E}_{i}$ which are associated with the equilibrium price $p(i=1,2)$. Thus

$$
W\left(\mathscr{E}_{i}, p\right)=\left\{f \in \mathscr{L}_{\varphi_{i}} \mid \int_{A_{i}} f \mathrm{~d} v_{i}=\int \boldsymbol{e} \circ \mathscr{E}_{i} \mathrm{~d} v_{i}\right\},
$$

where $\mathscr{L}_{\phi_{i}}$ denotes the set of all integrable selections of the correspondence $\varphi_{i}$ of $\left(A_{i}, \mathscr{A}_{i}, v_{i}\right)$ into $R^{l} ; a \mapsto \varphi\left(\mathscr{E}_{i}(a), p\right)(i=1,2)$.

In order to prove Theorem 1 it suffices to show that

$$
\overline{\mathscr{D} W\left(\mathscr{E}_{1}, p\right)}=\overline{\mathscr{D} W\left(\mathscr{E}_{2}, p\right)} \text {. }
$$

But this follows immediately from Theorem 3 in Hart-Kohlberg (1974). Indeed, the two correspondences $\varphi_{1}$ and $\varphi_{2}$ are integrably bounded since $p \gg 0$ and since $e \circ \mathscr{E}_{i}$ is integrable by assumption. Clearly $\varphi_{i}$ is closed-valued. It is not hard to show that $\varphi_{i}$ has a measurable graph [e.g., use D.II.3., Proposition 1.(b), and chapter 1.2., proposition 2 in Hildenbrand (1974)]. Finally, $\varphi_{1}$ and $\varphi_{2}$ are equally distributed. Indeed, for every Borel set $S$ in $R^{l}$ we have

$$
\begin{aligned}
v_{1}\left(\varphi_{1}^{-1}(S)\right)= & v_{1}\left(\mathscr{E}_{1}^{-1}\{(\precsim, e) \mid \varphi(\precsim, e, p) \cap S \neq \emptyset\}\right)= \\
& v_{2}\left(\mathscr{E}_{2}^{-1}\{(\precsim, e) \mid \varphi(\precsim, e, p) \cap S \neq \emptyset\}\right)=v_{2}\left(\varphi_{2}^{-1}(S)\right),
\end{aligned}
$$

since, by assumption, $\mathscr{E}_{1}$ and $\mathscr{E}_{2}$ are equally distributed. Hence all assumptions of Theorem 3 in Hart-Kohlberg (1974) are fulfilled. This completes the proof of Theorem $1 . \quad$ Q.E.D. 


\section{Standard representation}

The natural question to ask now is whether there are economies for which the set $\mathscr{D} W(\mathscr{E})$ is closed. The example given above shows that it does not suffice that there are many agents, i.e., that the economy is atomless. What one needs actually is that there are many agents of every characteristic in support of the measure $\mu$.

Let $\mu$ be a distribution of agents' characteristics. Then we define an economy $\mathscr{E}^{\mu}$ with distribution $\mu$, which we shall call the standard representation of $\mu$; the atomless measure space of agents is given by $A=\left(\mathscr{P}_{\mathrm{mo}} \times R_{+}^{l}\right) \times[0,1]$ with the product measure $\nu=\mu \otimes \lambda$, where $\lambda$ is the Lebesgue measure on $[0,1]$. The mapping $\mathscr{E}^{\mu}$ is simply the projection, i.e., $\mathscr{E}^{\mu}(\precsim, e, \xi)=(\precsim, e)$ for every $(\precsim, e, \xi) \in \mathscr{P}_{\mathrm{mo}} \times R_{+}^{l} \times[0,1]$.

With this notation we can state the following result:

Theorem 2. Let $\mu$ be a measure on $\mathscr{P}_{\mathrm{mo}} \times R_{+}^{l}$ with $\int e \mathrm{~d} \mu \gg 0$. Then $\mathscr{D} W\left(\mathscr{E}^{\mu}\right)$ is closed.

Proof. We give a proof under the additional assumption that the measure $\mu$ is concentrated on a compact set. The reader familiar with the concept of tightness will easily see that this assumption is not needed. Let $f_{n} \in W\left(\mathscr{E}^{\mu}\right)$ and assume that the sequence $\left(\mathscr{D} f_{n}\right)$ of distributions converges say $\mathscr{D} f_{n} \rightarrow \delta$. We have to show that there exists $f \in W\left(\mathscr{E}^{\mu}\right)$ with distribution $\mathscr{D} f=\delta$. Consider the joint distribution $\tau_{n}$ of the mapping $\left(\mathscr{E}^{\mu}, f_{n}\right)(n=1, \ldots)$. All mappings $\left(\mathscr{E}^{\mu}, f_{n}\right)$ take values in a compact subset of $\left(\mathscr{P}_{\mathrm{mo}} \times R^{l}\right) \times R^{l}$. Indeed, since the set $\Pi\left(\mathscr{E}^{\mu}\right)$ is closed and strictly positive and since by assumption all endowment vectors belong to a compact set, it follows that the sequence $\left(f_{n}\right)$ is bounded. It is well known that the set of probability measures on a compact metric space is weakly compact. Therefore we can assume without loss of generality that the sequence $\left(\tau_{n}\right)$ is weakly convergent, say $\tau_{n} \rightarrow \tau$. Note that the marginal distributions of $\tau$ on $\mathscr{P}_{\mathrm{mo}} \times R_{+}^{l}$ and $R_{+}^{l}$ are $\mu$ and $\delta$, respectively.

We now apply Skorokhod's Theorem [see e.g., Hildenbrand (1974, D.1)] to the sequence $\tau_{n} \rightarrow \tau$. Thus there is a measure space $(A, \mathscr{A}, v)$ and measurable mappings $\left(\mathscr{E}_{n}, \tilde{f}_{n}\right)(n=1,2, \ldots)$ and $(\widetilde{E}, \tilde{f})$ of $A$ into $\left(\mathscr{P}_{\text {mo }} \times R_{+}^{l}\right) \times R_{+}^{l}$ such that $\left(\mathscr{E}_{n}, \tilde{f}_{n}\right) \rightarrow(\mathscr{E}, \tilde{f})$ a.e. in $A$, and the distributions of $\left(\widetilde{\mathscr{E}}_{n}, \tilde{f}_{n}\right)$ and $(\mathscr{E}, f)$ are $\tau_{n}$ and $\tau$, respectively. Since $\left(\mathscr{E}^{\mu}, f_{n}\right)$ and $\left(\mathscr{E}_{n}, \tilde{f}_{n}\right)$ have the same joint distribution $\tau_{n}$, and since $f_{n} \in W\left(\mathscr{E}^{\mu}\right)$, it follows that $\tilde{f}_{n} \in W\left(\tilde{\mathscr{E}}_{n}\right)(n=1, \ldots)$. But this, together with the pointwise convergence of the sequence $\left(\tilde{E}_{n}, \tilde{f}_{n}\right)$ to $(\widetilde{E}, \tilde{f})$ implies by standard arguments that $\tilde{f} \in W(\widetilde{\mathscr{E}})$. Therefore, in order to complete the proof we have to show that there is an allocation $f$ for the economy $\mathscr{E}^{\mu}$ such that the joint distribution of $\left(\mathscr{E}^{\mu}, f\right)$ is equal to $\tau$. Indeed, it then follows that $f \in W\left(\mathscr{E}^{\mu}\right)$ and $\mathscr{D} f=\delta$ since $\tilde{f} \in W(\mathscr{E})$, and the joint distribution of $(\mathscr{E}, \tilde{f})$ is equal to $\tau$. Recall that the marginal distributions of $\tau$ are $\mu$ and $\delta$, respectively. 
Thus we have to construct ${ }^{2}$ a measurable mapping of the standard representation $\left(\mathscr{P}_{\mathrm{mo}} \times R_{+}^{l}\right) \times[0,1]$ into $\left(\mathscr{P}_{\mathrm{mo}} \times R_{+}^{l}\right) \times R_{+}^{l}$ whose distribution is $\tau$ and which is of the special form:

$$
(t, \xi) \mapsto(t, f(t, \xi))
$$

for every $t \in \mathscr{P}_{\mathrm{mo}} \times R_{+}^{l}$ and $\xi \in[0,1]$.

This mapping is obtained in the following way. Given the measure $\tau$ on the product $\left(\mathscr{P}_{\text {mo }} \times R_{+}^{l}\right) \times R_{+}^{l}$, let $\delta_{t}$ denote the (regular) conditional probability distribution for $(t, \xi) \mapsto \xi$ given $t$; thus $\delta_{t}$ is a probability measure on $R_{+}^{l}$, the mapping $t \mapsto \delta_{t}(V)$ is measurable for every Borel set $V$ in $R_{+}^{l}$ and one has

$$
\tau(U \times V)=\int_{U} \delta_{t}(V) \mu(\mathrm{d} t)
$$

for Borel sets $U$ in $\mathscr{P}_{\text {mo }} \times R_{+}^{l}$ and $V$ in $R_{+}^{l}$ [see e.g., Loève (1963, sections 26 and 27)].

Every measure $\delta_{t}$ on $R_{+}^{l}$ can be obtained as the image of the Lebesgue measure of a mapping $f(t, \cdot):[0,1] \rightarrow R_{+}^{l}$. Then we define the mapping $(t, \xi) \mapsto(t, f(t, \xi))$ which clearly has the distribution $\tau$. The functions $f(t, \cdot)$ can be chosen such that the function $f(\cdot, \cdot)$ is measurable. Indeed, by the wellknown Isomorphism Theorem we can assume without loss of generality that the measures $\delta_{t}$ are defined on $[0,1]$. Consider then the (accumulative) distribution function $F(t, \cdot)$ of $\delta_{t}$, i.e., $F(t, \xi)=\delta_{t}[0, \xi]$. Thus $F(t, \cdot)$ is a right-continuous function of $[0,1]$ into $[0,1]$ for every $t$, and $F(\cdot, \xi)$ is measurable for every $\xi$. This implies (see the lemma, below) that the function $F(\cdot, \cdot)$ is measurable. Hence, by choosing the function $f(t, \cdot)$ to be the reflexion on the diagonal of $F(t, \cdot)$, i.e.,

$$
f(t, \xi)= \begin{cases}0 & \text { if } \xi<F(t, 0) \\ \sup \{\eta \mid F(t, \eta) \leqq \xi\} & \text { otherwise }\end{cases}
$$

one obtains a function $f(\cdot, \cdot)$, which is measurable. Q.E.D.

Lemma. Let $(T, \mathscr{J})$ be a measurable space and $f$ a function of $T \times[0,1]$ into $[0,1]$ such that

(a) $f(\cdot, \xi)$ is $\mathscr{J}$-measurable for every $\xi \in[0,1]$;

(b) $f(t, \cdot)$ is right continuous for every $t \in T$.

Then the function $f$ is $\mathscr{J} \otimes \mathscr{B}[0,1]$-measurable.

Proof. For every integer $n=1, \ldots$ we define the function $f_{n}$ of $T \times[0,1]$ into $[0,1]$ by

$$
f_{n}(t, \xi)=f\left(t, \frac{k}{n}\right), \quad \text { if } \quad \frac{k-1}{n} \leq \xi<\frac{k}{n} \quad(k=1, \ldots, n)
$$

${ }^{2}$ We learned from David Freedman that constructions of this type belong to the folklore in Probability Theory. Since we could find no reference we thought it might be helpful to some readers if we outline the construction. 


$$
f_{n}(t, 1)=f(t, 1) \text {. }
$$

Clearly, by assumption (a) the function $f_{n}$ restricted to $T \times[(k-1) / n, k / n]$ $(k=1, \ldots, n)$ is measurable, and hence $f_{n}$ is measurable. By assumption (b) we have $\lim _{n} f_{n}(t, \xi)=f(t, \xi)$ for every $(t, \xi)$. Hence $f$ is measurable. Q.E.D.

\section{Upper hemicontinuity of Walras allocations in distribution}

For every distribution $\mu$ of agents' characteristics we define

$$
\mathscr{D} W(\mu)=\mathscr{D} W\left(\mathscr{E}^{\mu}\right) \text {. }
$$

Of course, we could have chosen any atomless economy $\mathscr{E}$ with distribution $\mu$ and define

$$
\mathscr{D} W(\mu)=\overline{\mathscr{D} W(\mathscr{E})}
$$

Theorem 3. Let $T$ be a compact subset in $\mathscr{P}_{\mathrm{mo}} \times R_{+}^{l}$. Then the correspondence $\mu \mapsto \mathscr{D} W(\mu)$ defined on the set of distribution on $T$ is compact-valued and u.h.c. at every distribution $\mu$ with strictly positive mean endowments $\int e \mathrm{~d} \mu \gg 0$.

Proof. First we remark that $\mathscr{D} W(\mu) \neq \emptyset$. Let the sequence $\left(\mu_{n}\right)$ converge to $\mu$ and let $\delta_{n} \in \mathscr{D} W\left(\mu_{n}\right)$. We have to show that there is a weakly converging subsequence $\left(\delta_{n_{q}}\right)$ whose limit $\delta$ belongs to $\mathscr{D} W(\mu)$. Since $\delta_{n} \in \mathscr{D} W\left(\mu_{n}\right)$ there exist a Walras allocation $f_{n}$ and a corresponding equilibrium price $p_{n}$ for the standard representation $\mathscr{E}^{\mu_{n}}$ such that $\mathscr{D} f_{n}=\delta_{n}$. It is not difficult to show [or, see Hildenbrand (1974, chapter 2, proposition 6)] that every limit point $p$ of the sequence $\left(p_{n}\right)$ is strictly positive. This implies that the sequence $\left(f_{n}\right)$ is bounded. Now consider the joint distribution $\tau_{n}$ of the mapping $\left(\mathscr{E}^{\mu_{n}}, f_{n}\right)$. The sequence $\left(\tau_{n}\right)$ is contained in a compact set, and, therefore, we can assume without loss of generality that the sequence $\left(\tau_{n}\right)$ is convergent, say $\tau_{n} \rightarrow \tau$. Using Skorokhod's Theorcm as in the proof of Theorem 2 one shows that there is an atomless economy $\mathscr{E}$ and a Walras allocation $f \in W(\mathscr{E})$ such that the distribution of $(\mathscr{E}, f)$ is equal to $\tau$. Since the marginal distributions of $\tau$ are $\mu$ and $\delta$, respectively, it follows that $\delta \in \mathscr{D} W(\mathscr{E})$, and hence $\delta \in \mathscr{D} W(\mu)$. Q.E.D.

\section{References}

Aumann, R.J., 1964, Markets with a continuum of traders, Econometrica 32, 39-50.

Aumann, R.J., 1966, Existence of competitive equilibria in markets with a continuum of traders, Econometrica 34, 1-17.

Hart, S. and E. Kohlberg, 1974, Equally distributed correspondences, Journal of Mathematical Economics, this issue.

Hildenbrand, W., 1970, On economies with many agents, J. Econ. Theory 2, 161-188.

Hildenbrand, W., 1974, Core and equilibria of a large economy (Princeton University Press, Princeton) to appear.

Kannai, Y., 1970, Continuity properties of the core of a market, Econometrica 38, 791-815.

Loève, M., 1963, Probability theory, 3rd ed. (Van Nostrand, Princeton, N.J.). 